\title{
GMRT observations of extragalactic radio sources with steeply inverted spectra
}

\author{
Mukul Mhaskey $^{1 \star}$, Gopal-Krishna ${ }^{2}$, Pratik Dabhade ${ }^{3,4}$, Surajit Paul ${ }^{1}$, \\ Sameer Salunkhe ${ }^{1}$ and S.K. Sirothia ${ }^{5,6}$ \\ ${ }^{1}$ Department of Physics, Savitribai Phule Pune Unversity, Ganeshkhind, Pune 411007, India \\ ${ }^{2}$ Aryabhatta Research Institute of Observational Sciences (ARIES), Manora Peak, Nainital - 263129, India \\ ${ }^{3}$ Inter University Centre for Astronomy and Astrophysics (IUCAA), Pune 411007, India \\ ${ }^{4}$ Leiden Observatory, Leiden University, Niels Bohrweg 2, 2333 CA, Leiden, Netherlands \\ ${ }^{5}$ Square Kilometre Array South Africa, 3rd Floor, The Park, Park Road, Pinelands, 7405, South Africa \\ ${ }^{6}$ Department of Physics and Electronics, Rhodes University, PO Box 94, Grahamstown, 6140, South Africa
}

1 February 2019

\begin{abstract}
We report quasi-simultaneous GMRT observations of seven extragalactic radio sources at $150,325,610$ and $1400 \mathrm{MHz}$, in an attempt to accurately define their radio continuum spectra, particularly at frequencies below the observed spectral turnover. We had previously identified these sources as candidates for a sharply inverted integrated radio spectrum whose slope is close to, or even exceeds $\alpha_{c}=+2.5$, the theoretical limit due to synchrotron self-absorption (SSA) in a source of incoherent synchrotron radiation arising from relativistic particles with the canonical (i.e., power-law) energy distribution. We find that four out of the seven candidates have an inverted radio spectrum with a slope close to or exceeding +2.0 , while the critical spectral slope $\alpha_{c}$ is exceeded in at least one case. These sources, together with another one or two reported in very recent literature, may well be the archetypes of an extremely rare class, from the standpoint of violation of the SSA limit in compact extragalactic radio sources. However, the alternative possibility that free-free absorption is responsible for their ultra-sharp spectral turnover cannot yet be discounted.
\end{abstract}

Key words: radiation mechanisms: non thermal - galaxies: ISM - galaxies: jets galaxies: nuclei - quasars: general - radio continuum: galaxies

\section{INTRODUCTION}

A few years ago we initiated a targeted search for 'Extremely Inverted Spectrum Extragalactic Radio Sources'(EISERS) characterized by an integrated radio spectrum which turns over, attaining a slope $\alpha$ that exceeds $\alpha_{c}=+2.5$ (GopalKrishna et al. 2014); (hereafter Paper I). This critical value, $\alpha_{c}$ is important as it represents the theoretical limit which can be achieved (via self-absorption) for an source of incoherent synchrotron radiation arising from a power-law energy distribution of relativistic charged particles, which is the basic mechanism widely held responsible for the radio continuum emission from active galactic nuclei (Slish 1963; Scheuer \& Williams 1968; Rybicki \& Lightman 1986). The Synchrotron Self-Absorption (SSA) can also produce shallower spectral gradient below the spectral turnover, as witnessed in many extragalactic radio sources, but this can be readily understood in terms of inhomogeneity within the

* E-mail:mhaskeymukul@gmail.com

(C) 2018 The Authors

\footnotetext{
C 2018 The Authors
}

source region contributing to the emission below the spectral turnover (O'Dea 1998; Tingay \& de Kool 2003). The basic physics underlying the SSA limit is that the source cannot have a brightness temperature in excess of the plasma temperature of the incoherently radiating non-thermal electrons (Kellermann \& Pauliny-Toth 1969; Pacholczyk 1970). Discovery of rare radio galaxies with $\alpha>+2.5$ may then call for an explanation other than the SSA. One possibility is that free-free absorption (FFA) due to clouds/screen of thermal plasma is causing the sharp inversion of the integrated radio spectrum (Kellermann 1966; Kuncic et al. 1998; Kameno et al. 2000; Vermeulen et al. 2003). Different versions of this basic scenario have been summarized by Callingham et al. (2015). Thus, FFA could occur in gas clouds of the narrowline regions (NLR) engulfed by the radio lobes. AGN radiation and the synchrotron UV emission from the lobes could keep the compressed surface layers of the NLR clouds photoionized (Stawarz et al. 2008; Ostorero et al. 2010). The thermal gas clouds could also be photo-ionised by the bow shock associated with the jet propagating in the external medium 
(van Breugel 1984; Bicknell et al. 1997, 2018). As mentioned in Paper I, FFA effects (leading to $\alpha>+2.5$ ) have indeed been observed in a few radio galaxies, albeit only for their parsec-scale nuclear radio jets. Prominent examples include the well-known radio source 3C 345 (Matveenko et al. 1990), Centaurus A (Jones et al. 1996; Tingay \& Murphy 2001), Cygnus A (Krichbaum et al. 1998), NGC 1275/Perseus A (Levinson et al. 1995; Walker et al. 2000), NGC 4261 (Jones et al. 2001) and NGC 1052 (Vermeulen et al. 2003; Kadler et al. 2004).

An alternative scenario for the putative EISERS $(\alpha>$ +2.5 ), perhaps more salient from the perspective of AGN physics, would be that in some rare sources, the low-energy spectrum of the radiating leptons itself differs from the canonical power-law shape, for example, being either monoenergetic, or a Maxwellian (Rees 1967), or it has a large leptonic excess at low energies, over a power-law distribution (de Kool \& Begelman 1989). On the observational side, only a few reports had existed of radio galaxies with integrated spectrum exhibiting a slope which approaches even +2.0 . For instance, during a huge radio flare, the blazar-like spiral galaxy III Zw 2 exhibited an inverted spectrum with $\alpha=+1.9 \pm 0.1$ (Falcke et al. 1999). Another few possible examples showing sharply inverted integrated radio spectra are reported by Murphy et al. (2010) see, also (Dallacasa et al. 2000). But, since the SSA limit was not violated in any of these cases, this motivated us to initiate a systematic search for more extreme cases of inverted radio spectrum (Paper I).

It may be noted that, as compared to the opaque part of the radio spectrum, the origin of ultra-steep spectrum in the optically thin spectral regime has been discussed in the literature much more extensively. Such sources (having $\alpha<-1.0)$ are commonly referred to as "Ultra-Steep Spectrum " (USS) radio sources. With the increased availability of measurements below $0.5 \mathrm{GHz}$, several of them have, in fact, been reclassified as Gigahertz-Peaked-Spectrum (GPS) sources (e.g. Callingham et al. 2017, and references therein), or even Megahertz-Peaked-Spectrum (MPS) sources (Falcke et al. 2004; Coppejans et al. 2015, 2016). It has been suggested (Callingham et al. 2017; Murgia et al. 2002) that in the case of USS sources, the low-frequency part of the radio spectrum, which is supposed to manifest the particle injection spectrum, remains essentially unobserved since it has already migrated either to frequencies that lie in the optically thick spectral regime, or even outside the normally accessed radio window. In this scenario, the observed ultrasteep spectrum in the optically thin regime is actually the radiatively steepened part of the radio spectrum, as expected from ageing of relativistic electrons in the continuous injection model (Kardashev 1962; Kellermann 1964). This interpretation of the USS spectrum being an ageing effect would also be consistent with the empirically established anticorrelation, between the radio spectral curvature and the steepness of the spectral slope measured, for classical double radio sources (say near $1 \mathrm{GHz}$ in the rest-frame) (Mangalam \& Gopal-Krishna 1995). The same anti-correlation is, in fact, reflected in the observed straightness of the radio spectra of the USS sources, as highlighted by Klamer et al. (2006). An alternative scenario to this which associates ultra-steep spectrum with aged but still active radio sources (e.g. Callingham et al. 2017; Mangalam \& Gopal-Krishna
1995), posits that the USS sources are young and reside in dense environments (which is expected to be more common at high redshifts). As a result, the advance of their hot spots is slowed down, giving rise to a steep energy spectrum of the relativistic electrons accelerated there via the firstorder Fermi process, which causes an ultra-steep straight synchrotron radio spectrum ab initio (Athreya \& Kapahi 1998; Klamer et al. 2006; Bicknell et al. 1997). This potentially attractive theoretical scenario would, however, need further substantiation, particularly in view of the empirical study presented by Gopal-Krishna et al. (2012), which found no correlation of the particle injection spectral index with rotation measure for powerful double radio sources.

In section 2 we describe the selection procedure for the seven EISERS candidates. Section 3 contains the details of the quasi-simultaneous radio observations and the data analysis procedure. Notes on individual sources are given in section 4 . This is followed by a brief discussion on the EISERS and the conclusions, in sections $5 \& 6$ respectively.

\section{SAMPLE DEFINITION}

As a first step, we reported in Paper I a set of 7 sources for which we had estimated an $\alpha>+2.0$, by comparing the TIFR.GMRT.SKY.Survey (TGSS/DR5) made at 150 $\mathrm{MHz}$ using the Giant Metrewave Radio Telescope (GMRT) (Swarup 1991) and the 'Westerbork In the Southern Hemisphere '(WISH) survey at $352 \mathrm{MHz}$ (De Breuck et al. 2002). At that time these two surveys were the deepest available large-area sky surveys at metre wavelengths, with arcminute or better resolution and a typical rms noise under $10 \mathrm{mJy} /$ beam. Focussing attention only on the 7056 sources within the region of overlap between these two surveys, which had been listed as type ' $\mathrm{S}$ '(i.e. single component) sources with flux densities above $100 \mathrm{mJy}$ at $352 \mathrm{MHz}$ in the WISH catalogue, we found seven sources having $\alpha$ (150$352 \mathrm{MHz})>+2.0$ (Paper I). Two of them were actually undetected at $150 \mathrm{MHz}$ and hence only lower limits could be set to the slopes of their inverted spectra. All the seven sources appear unresolved in their TGSS images and a GPS type peaked radio spectrum could be confirmed for at least 5 of the 7 sources, based on the existing radio measurements. ${ }^{1}$.

In Paper I we underlined two of the seven sources as highly promising EISERS candidates, since their nondetection in the TGSS at $150 \mathrm{MHz}$ implied $\alpha$ (150-352 MHz) $>+2.35$. Soon thereafter, Callingham et al. (2015) reported discovery of a very interesting 'extreme GPS source' PKS B0008-421, based on an exceptionally dense spectral sampling between 0.1 and $22 \mathrm{GHz}$, covering comprehensively both sides of the single spectral peak seen near 0.6 GHz. Strikingly, its spectral slope is seen to become as large as +2.4 below the turnover frequency, closely approaching the SSA limit of $\alpha_{c}=+2.5$. Although, the multi-frequency measurements used by them are non-contemporaneous, the authors argued that any flux variability should be too small to have significantly affected the radio spectrum below the

1 GPS sources are defined as having an integrated spectrum that shows a single peak and steep slopes on either side of the peak, cf. (Spoelstra et al. 1985; Gopal-Krishna et al. 1983; Gopal-Krishna \& Spoelstra 1993; O'Dea 1998; An \& Baan 2012) 
Inverted spectrum extragalactic radio sources

turnover. Based on physical considerations, they showed a slight preference for an inhomogeneous FFA model, over the SSA model, as being the dominant cause of the sharp spectral turnover observed. In a follow-up paper, Callingham et al. (2017) (hereafter CEG17) have reported a few more examples of such sources, which are briefly discussed in Sect. 5.

Clearly, the results presented in Paper I were only meant to be an initial step towards a systematic search for EISERS $(\alpha>+2.5)$, particularly in view of the following two caveats mentioned there: Firstly, the two radio surveys (TGSS/DR5 and WISH) used for computing the spectral indices of individual sources were made at epochs nearly a decade apart. The long time interval could then have introduced significant uncertainty due to flux variability expected from refractive interstellar scintillation at such low frequencies (Bell et al. 2019). The second caveat is that since the overlap region between the two surveys lies at fairly low declinations $(-10>\delta>-30 \mathrm{deg})$, the north-south beamwidth of the $352 \mathrm{MHz}$ WISH survey is rather large (FWHM $=0.9$ cosec $\delta \sim 2$ arcmin). The enhanced confusion could then have significantly impacted the accuracy of flux measurement. It may also be noted that even the WENSS (Rengelink et al. 1997), which is the northern-sky counterpart of WISH and therefore much less affected by the beam elongation, is known to be off the Baars et al. (1977) flux scale or in the flux scale of Roger, Costain \& Bridle, (RCB, Roger et al. 1973) by over $10 \%$ (see Hardcastle et al. 2016). Therefore, the combined uncertainty associated with the measurement and calibration of the WISH and TGSS-DR5 flux densities could have biased the previous estimates of spectral index. This also explains why the WISH flux densities are often found to be significantly off from the GMRT measurements at $325 \mathrm{MHz}$ (Section 4). Rectifying both these shortcomings, we present here previously unpublished GMRT observations of all the seven EISERS candidates reported in $\mathrm{Pa}$ per I. These observations, made at 150, 325, 610 and 1400 $\mathrm{MHz}$, have the highest sensitivity and resolution currently available for these sources at such low frequencies and, furthermore, they are nearly contemporaneous, all having been carried out within a time span of just four weeks ( 3 weeks between the observations at the lowest two frequencies, namely 150 and $325 \mathrm{MHz}$ ). We further note that the present observations have provided for each source at least two well-spaced data points below the spectral peak, thus enabling a better constrained spectral turnover than is the norm for GPS sources, a point also highlighted in CEG17.

\section{RADIO OBSERVATION AND ANALYSIS}

\subsection{Radio Observations}

The 7 EISERS candidates were observed with the GMRT (Swarup 1991) in the snapshot mode, quasi-simultaneously at 150, 325, 610 and $1400 \mathrm{MHz}$, between 2014 July 09 and 2014 August 05 (Table 1). The integration time was $2 \mathrm{sec}$ at $150 \mathrm{MHz}$ (bandwidth $16 \mathrm{MHz}$ ) and $16 \mathrm{sec}$ at the remaining 3 frequencies (bandwidth $32 \mathrm{MHz}$ ). One flux calibrator, out of $3 \mathrm{C} 286,3 \mathrm{C} 48$, and $3 \mathrm{C} 147$, was observed at the start and the end of each observing session. Their flux densities were taken from the VLA calibrator manual (Perley \& Butler 2017). Each snapshot of a given target source was sandwiched between a pair of snapshots on its phase calibrator(s). The average total on-target time depends on frequency, being $\sim 40$ minutes at $150 \mathrm{MHz}$ and as short as 2 minutes at $1400 \mathrm{MHz}$. Table 1 contains the observation log providing additional details for the different observing frequencies. Table 2 lists flux density measurements at different frequencies, taken from the present GMRT observations and from the literature. The radio contour maps at $150 \mathrm{MHz}$ and $325 \mathrm{MHz}$ for the sources J0242-1649 and J1209-2032 are presented in Figures $1 \& 2$, respectively.

\subsection{Analysis}

The astronomical packages SPAM and AIPS were used for the data analysis. The visibilities at the low frequencies of $150 \mathrm{MHz}$ and $325 \mathrm{MHz}$ were processed using the SPAM (Source Peeling and Atmospheric Modelling; Intema (2014)) package, which is a semi-automated pipeline based on AIPS, Parseltongue and Python. SPAM performs a series of iterative flagging and calibration and the imaging is done with direction dependent calibration. This package has recently been used by Intema et al. (2017) for processing the entire TIFR-GMRT SKY SURVEY (TGSS) data at $150 \mathrm{MHz}$. Details of SPAM and its routines are provided by Intema et al. (2017)

The $610 \mathrm{MHz}$ and $1400 \mathrm{MHz}$ GMRT observations were reduced using the Astronomical Image Processing System (AIPS). The standard procedure consisted of first cleaning up the data for RFI (bad visibility data points) using the 'flgit' and 'uvflg' tasks, followed by the flux and phase calibration protocols. The Perley-Butler (Perley \& Butler 2017) absolute flux density scale was used to set the flux densities of the flux calibrators, which in turn were used to define the flux scale for the respective phase calibrators and the target sources. Calibrated visibilities were transformed into radio image (deconvolved images) using the 'IMAGR' task which performs the self-calibration for mitigating the antennabased phase and amplitude errors. Several rounds of phaseonly self-calibration cycles were carried out, concluded by one round of amplitude plus phase calibration. Lastly, a correction for the antenna primary beam was applied to the map obtained after the final self-calibration round. The flux density uncertainties of individual sources have been computed using the expression given in equation 1 , wherein the $10 \%$ of the peak flux density accounts for the systematic error component, including the small errors arising from the gain dependence of the antennas on their elevation angle changes (Chandra et al. 2004).

$$
\sqrt{(\text { map rms })^{2}+(10 \% \text { of the peak flux })^{2}}
$$

As a cautionary check on possible systematic bias in the flux density scales of the present $150 \mathrm{MHz}$ GMRT maps, we have additionally determined the scaling factors by comparing the present map of each target field with its counterparts in the TGSS-ADR1 (Intema et al. 2017) and/or GLEAM surveys (Hurley-Walker et al. 2017), also made at $150 \mathrm{MHz}$. For a given GMRT map (measuring $1 \times 1 \mathrm{deg}$ ), the flux scaling factor (FSF) was determined by taking average of the ratios of the measured flux densities of several discrete sources in the present $150 \mathrm{MHz}$ GMRT map and their coun- 
Table 1. GMRT observation log and the map parameters.

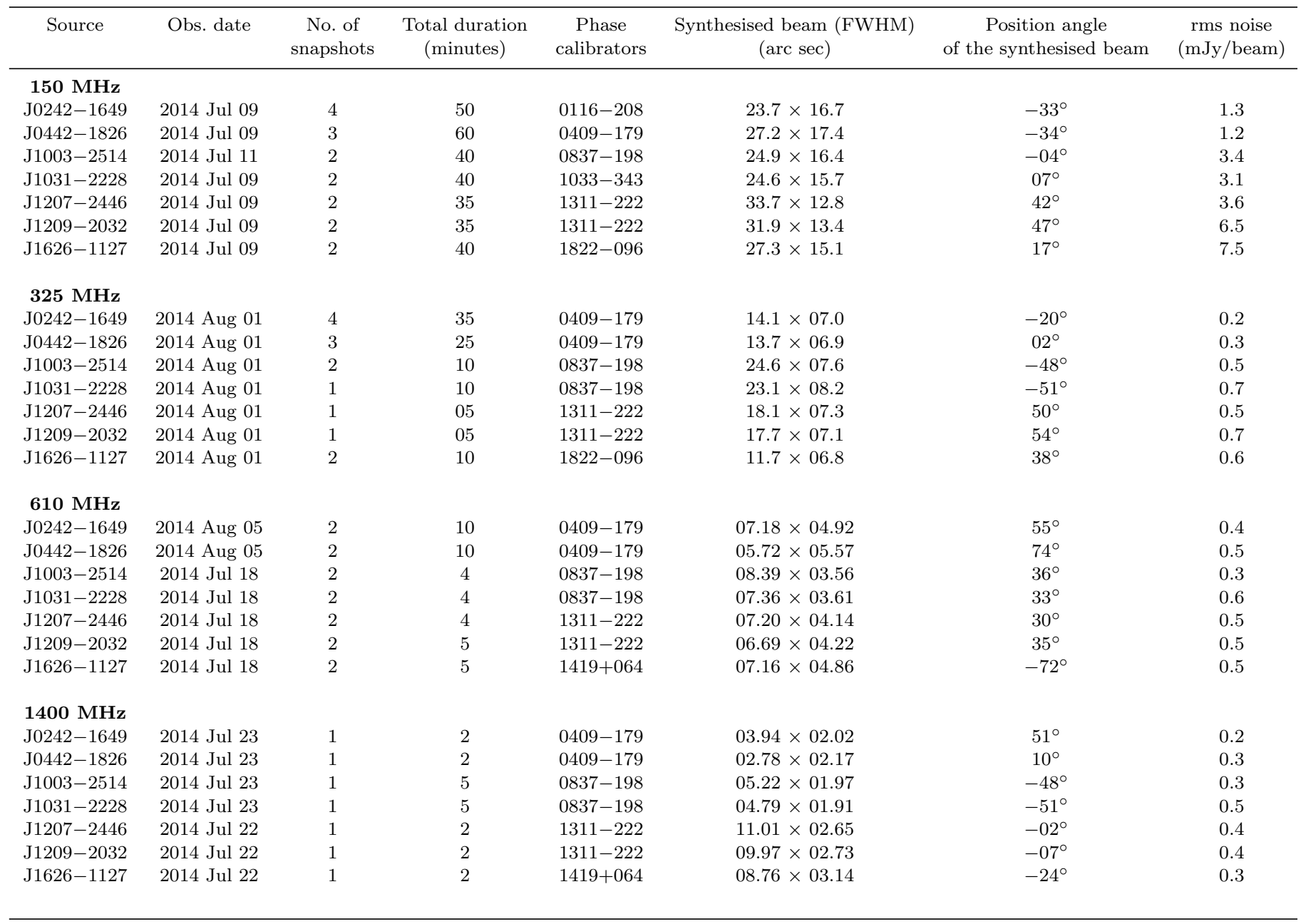

Table 2. Positions (J2000), flux densities and spectral indices of the 7 EISERS candidates.

\begin{tabular}{|c|c|c|c|c|c|c|c|c|c|c|c|c|c|}
\hline $\begin{array}{l}\text { Source position } \\
\text { GMRT }^{\star} \\
\text { at } 325 \mathrm{MHz}\end{array}$ & $\begin{array}{c}150 \mathrm{MHz} \\
\text { GMRT } \\
(\mathrm{mJy})\end{array}$ & $\begin{array}{c}325 \mathrm{MHz} \\
\text { GMRT } \\
(\mathrm{mJy})\end{array}$ & $\begin{array}{c}610 \mathrm{MHz} \\
\text { GMRT } \\
(\mathrm{mJy})\end{array}$ & $\begin{array}{c}1400 \mathrm{MHz} \\
\text { GMRT } \\
(\mathrm{mJy})\end{array}$ & $\begin{array}{c}150 \mathrm{MHz} \\
\text { TGSS-ADR } 1^{\star} \\
(\mathrm{mJy})\end{array}$ & $\begin{array}{c}151 \mathrm{MHz} \\
\text { GLEAM }^{\star} \\
(\mathrm{mJy})\end{array}$ & $\begin{array}{c}352 \mathrm{MHz}^{\star} \\
\text { WISH }^{\star} \\
(\mathrm{mJy})\end{array}$ & $\begin{array}{c}1400 \mathrm{MHz}^{\mathrm{NVS}} \\
(\mathrm{mJy})\end{array}$ & $\begin{array}{l}4.85 \mathrm{GHz}^{\star} \\
\mathrm{PMN}^{\star} \\
(\mathrm{mJy})\end{array}$ & $\begin{array}{c}4.85 \mathrm{GHz}^{\star} \\
\mathrm{ATCA}^{\star} \\
\left(\mathrm{mJy}^{2}\right)\end{array}$ & $\begin{array}{c}8 \mathrm{GHz} \\
\mathrm{ATCA}^{\star} \\
(\mathrm{mJy})\end{array}$ & $\begin{array}{l}20 \mathrm{GHz}^{\star} \\
\mathrm{ATCA}^{\star} \\
(\mathrm{mJy})\end{array}$ & $\begin{array}{l}\text { Spectral Index } \\
(150-352 \mathrm{MHz})\end{array}$ \\
\hline $\begin{array}{l}0242 \quad 10.57 \\
-16 \quad 4933.5\end{array}$ & $\begin{array}{c}6.4 \pm 1.4 \\
(1.3)\end{array}$ & $\begin{array}{c}62.7 \pm 6.3 \\
(0.2)\end{array}$ & $\begin{array}{c}167.2 \pm 16.7 \\
(0.4)\end{array}$ & $\begin{array}{l}75.2 \pm 7.5 \\
(0.2)\end{array}$ & $\begin{array}{l}<13 \\
(2.6)\end{array}$ & $\begin{array}{l}<54.9 \\
(18.3)\end{array}$ & $106 \pm 4.5$ & $96.7 \pm 2.9$ & & & & & $2.95 \pm 0.31$ \\
\hline $\begin{array}{l}0442 \quad 01.20 \\
-18 \quad 2634.0\end{array}$ & $\begin{array}{l}35.6 \pm 3.8 \\
\quad(1.3)\end{array}$ & $\begin{array}{l}88.2 \pm 8.8 \\
(0.3)\end{array}$ & $\begin{array}{l}105.8 \pm 10.6 \\
(0.5)\end{array}$ & $\begin{array}{l}43.5 \pm 4.5 \\
(0.3)\end{array}$ & $\begin{array}{l}<16 \\
(3.2)\end{array}$ & $\begin{array}{l}<73.2 \\
(24.4)\end{array}$ & $105 \pm 4.4$ & $50.9 \pm 1.6$ & $85 \pm 11$ & & & & $1.17 \pm 0.19$ \\
\hline $\begin{array}{llll}10 & 03 & 06.19 \\
-25 & 14 & 05.5\end{array}$ & $\begin{array}{c}9.4 \pm 3.5 \\
(3.4)\end{array}$ & $\begin{array}{l}47.9 \pm 4.8 \\
\quad(0.5)\end{array}$ & $\begin{array}{c}154.8 \pm 15.5 \\
(0.3)\end{array}$ & $\begin{array}{c}62.1 \pm 6.2 \\
(0.3)\end{array}$ & $\begin{array}{l}19.2 \pm 2.0 \\
\quad(4.1)\end{array}$ & $\begin{array}{c}37.5 \pm 20.6 \\
(20.8)\end{array}$ & $143 \pm 6.1$ & $74.1 \pm 2.8$ & & & & & $2.11 \pm 0.50$ \\
\hline $\begin{array}{l}103152.20 \\
-22 \quad 2826.2\end{array}$ & $\begin{array}{l}22.8 \pm 3.6 \\
\quad(3.1)\end{array}$ & $\begin{array}{l}107.1 \pm 10.7 \\
\quad(0.7)\end{array}$ & $\begin{array}{c}311.6 \pm 31.2 \\
(0.6)\end{array}$ & $\begin{array}{l}307.7 \pm 30.8 \\
\quad(0.5)\end{array}$ & $\begin{array}{l}56.1 \pm 9.5 \\
\quad(4.7)\end{array}$ & $\begin{array}{l}46.0 \pm 17.1 \\
\quad(20.3)\end{array}$ & $191 \pm 7.1$ & $371.4 \pm 11.2$ & $328 \pm 20$ & $371 \pm 19$ & $291 \pm 15$ & $124 \pm 8$ & $2.00 \pm 0.24$ \\
\hline 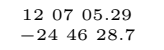 & & $\begin{array}{c}172.3 \pm 17.2 \\
(0.5)\end{array}$ & 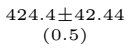 & $\begin{array}{l}205.2 \pm 20.52 \\
\quad(0.4)\end{array}$ & $\begin{array}{c}69.6 \pm 9.2 \\
(3.6)\end{array}$ & $\begin{array}{l}73.4 \pm 19.3 \\
\quad(22.6)\end{array}$ & $380 \pm 23$ & $226.7 \pm 6.8$ & $105 \pm 12$ & & & & $1.17 \pm 0.21^{\ddagger}$ \\
\hline $\begin{array}{l}120915.31^{\dagger} \\
-203234.4\end{array}$ & $\begin{array}{l}<19.5 \\
(6.5)\end{array}$ & $\begin{array}{l}149.4 \pm 14.9 \\
\quad(0.7)\end{array}$ & $\begin{array}{l}422.9 \pm 42.3 \\
(0.5)\end{array}$ & $\begin{array}{l}326.2 \pm 32.6 \\
\quad(0.4)\end{array}$ & $\begin{array}{l}<17.5 \\
(3.5)\end{array}$ & $\begin{array}{l}<64.5 \\
(21.5)\end{array}$ & $207 \pm 8.4$ & $353.7 \pm 10.6$ & $573 \pm 32$ & $832 \pm 42$ & $1177 \pm 61$ & $707 \pm 46$ & $>2.64 \pm 0.13$ \\
\hline $\begin{array}{lll}16 & 26 & 51.82 \\
-11 & 27 & 24.0\end{array}$ & $\begin{array}{c}37.8 \pm 8.4 \\
\quad(7.5)\end{array}$ & $\begin{array}{l}152.5 \pm 15.3 \\
(0.6)\end{array}$ & $\begin{array}{l}154.4 \pm 15.5 \\
(0.5)\end{array}$ & $\begin{array}{l}56.7 \pm 5.7 \\
(0.3)\end{array}$ & $\begin{array}{l}84.1 \pm 9.5 \\
\quad(3.3)\end{array}$ & $\begin{array}{c}65.1 \pm 41.4 \\
\quad(47.6)\end{array}$ & $206 \pm 8.5$ & $52.3 \pm 1.6$ & & & & & $1.80 \pm 0.32$ \\
\hline References & & $\begin{array}{l}\text { arup }(19 \\
\text { ragalact } \\
\text { that th }\end{array}$ & $\begin{array}{c}\text { 1); TGSS-ADR } \\
\text { Database; N } \\
\text { values given in } \\
+\mathrm{J} 120\end{array}$ & $\begin{array}{l}21-\text { Intema e } \\
\text { VSS - Condo } \\
\text { nside parent1 } \\
9-2032 \text { is id }\end{array}$ & $\begin{array}{l}\text { al. (2017); C } \\
\text { et al. (1998 } \\
\text { eses, just bel } \\
\text { ntified with }\end{array}$ & $\begin{array}{l}\text { EAM - Hurl } \\
\text { PMN - Griff } \\
\text { the flux de } \\
\text { galaxy at } z\end{array}$ & $\begin{array}{l}\text { ley-Walke } \\
\text { ffith et al } \\
\text { ensities, a } \\
=0.404\end{array}$ & $\begin{array}{l}\text { et al. (2017) } \\
\text { (1994); ATC } \\
\text { e the rms err } \\
\text { Healey et al. }\end{array}$ & $\begin{array}{l}\text {; WISH } \\
\text { A - Murp } \\
\text { rors of the } \\
2008)\end{array}$ & $\begin{array}{l}\text { De Breuc } \\
\text { hy et al. } \\
\text { respective }\end{array}$ & $\begin{array}{l}\text { et al. } \\
\text { 010). } \\
\text { maps. }\end{array}$ & & \\
\hline
\end{tabular}


Table 3. Flux-density scaling factors (FSFs) and the correspondingly adjusted values of $\alpha(150-325 \mathrm{MHz})$.

\begin{tabular}{|c|c|c|c|c|c|}
\hline Source & $\begin{array}{c}\text { FSF-1 } \\
\text { TGSS/GMRT }\end{array}$ & $\begin{array}{c}\text { FSF-2 } \\
\text { GLEAM/GMRT }\end{array}$ & $\alpha^{\dagger}$ & $\alpha_{T G S S}^{\ddagger}$ & $\alpha_{G L E A M}^{\ddagger}$ \\
\hline $\begin{array}{lll}02 & 42 & 10.57 \\
-16 & 49 & 33.5\end{array}$ & 0.95 & 0.83 & $2.95 \pm 0.31$ & $3.02 \pm 0.33$ & $3.19 \pm 0.37$ \\
\hline $\begin{array}{ccc}04 & 42 & 01.20 \\
-18 & 26 & 34.0\end{array}$ & 0.73 & 0.72 & $1.17 \pm 0.19$ & $1.58 \pm 0.19$ & $1.60 \pm 0.19$ \\
\hline $\begin{array}{llll}10 & 03 & 06.19 \\
-25 & 14 & 05.5\end{array}$ & 1.06 & 0.92 & $2.11 \pm 0.50$ & $2.03 \pm 0.48$ & $2.21 \pm 0.54$ \\
\hline $\begin{array}{l}103152.20 \\
-22 \quad 28 \quad 26.2\end{array}$ & 1.21 & 0.98 & $2.00 \pm 0.24$ & $1.75 \pm 0.24$ & $2.03 \pm 0.26$ \\
\hline $\begin{array}{lll}12 & 07 & 05.29 \\
-24 & 46 & 28.7\end{array}$ & - & - & $1.17 \pm 0.21^{\star}$ & - & - \\
\hline $\begin{array}{l}12 \quad 09 \quad 15.31 \\
-20 \quad 32 \quad 34.4\end{array}$ & 1.44 & 1.37 & $2.64 \pm 0.13$ & $2.16 \pm 0.39$ & $2.23 \pm 0.41$ \\
\hline $\begin{array}{l}162651.82 \\
-112724.0 \\
\end{array}$ & 1.26 & 0.97 & $1.80 \pm 0.32$ & $1.51 \pm 0.27$ & $1.84 \pm 0.32$ \\
\hline $\begin{array}{l}\dagger \\
\dagger \text { The present } \\
\text { is applied. Flu } \\
\text { GMRT maps } \\
\text { * The } 150 \mathrm{MH} \\
\text { as the present } \\
\ddagger \text { Estimate of }\end{array}$ & $\begin{array}{l}\text { estimate of spe } \\
\text { x densities at b } \\
\text { eported here, ex } \\
\text { Iz flux density } \\
\text { GMRT map is } \\
\text { spectral Index ( }\end{array}$ & $\begin{array}{l}\text { ctral Index }(150- \\
\text { oth } 150 \mathrm{MHz} \text { and } \\
\text { cept for J0242-1 } \\
\text { for this source ha } \\
\text { severely affected } \\
150-325 \mathrm{MHz} \text { ) wh }\end{array}$ & $\begin{array}{l}5 \mathrm{MHz} \text { ) whe } \\
25 \mathrm{MHz} \text { are } \\
19 \text { (see the fo } \\
\text { been taken } \\
\text { RFI. } \\
\text { scaling fact }\end{array}$ & $\begin{array}{l}\text { n no scaling } \\
\text { measured frc } \\
\text { otnote to Ta } \\
\text { from TGSS-. }\end{array}$ & $\begin{array}{l}\text { factor } \\
\text { om the } \\
\text { ble 2). } \\
\text { ADR1, }\end{array}$ \\
\hline
\end{tabular}

terparts in the other $150 \mathrm{MHz}$ map being compared. This was done after ensuring that the chosen sources are stronger than $\sim 100$ mJy and located in relatively isolated environment within the maps. Table 3 gives the so obtained values of FSF for each of our $150 \mathrm{MHz}$ GMRT map. Thus, multiplying the present $150 \mathrm{MHz}$ GMRT flux densities with the FSF value(s) estimated for that field translates the present flux densities to the flux scales of the TGSS-ADR 1 and the GLEAM survey. The correspondingly adjusted values of the spectral index $\alpha$ (150-325 MHz) of individual target sources are also listed in Table 3. We caution that the estimates of FSF have only been used for calculating the spectral indices $\alpha_{(1)} \& \alpha_{(2)}$ given in Table 3 and are not meant for general applicability. Figure 3 shows the radio spectra of the seven sources.

\section{NOTES ON INDIVIDUAL SOURCES}

\section{J0242-1649}

Although this source is not seen in the TGSS-ADR 1 and GLEAM maps, the high sensitivity of the present GMRT map $(\sigma=1.3 \mathrm{mJy}$, Figure 1$)$ has led to its detection at $150 \mathrm{MHz}$. Its flux densities at $150 \mathrm{MHz}$ and the 3 higher frequencies, measured in the present GMRT observations are given in Table 2, along with the same information for the remaining six of our target sources. The new GMRT maps at $150 \mathrm{MHz}$ and $325 \mathrm{MHz}$ for our most promising targets are shown in Figures 1 and 2 (the maps for the remaining sources are included as part of the online material). At 325 $\mathrm{MHz}$, although the source is clearly detected in the present map, its flux density is much lower $(\sim 60 \%)$ than that given in the WISH catalog at $352 \mathrm{MHz}$. Still, its inverted spectrum with $\alpha=+2.95 \pm 0.31$ remains steep enough to qualify it as an EISERS (Table 2).
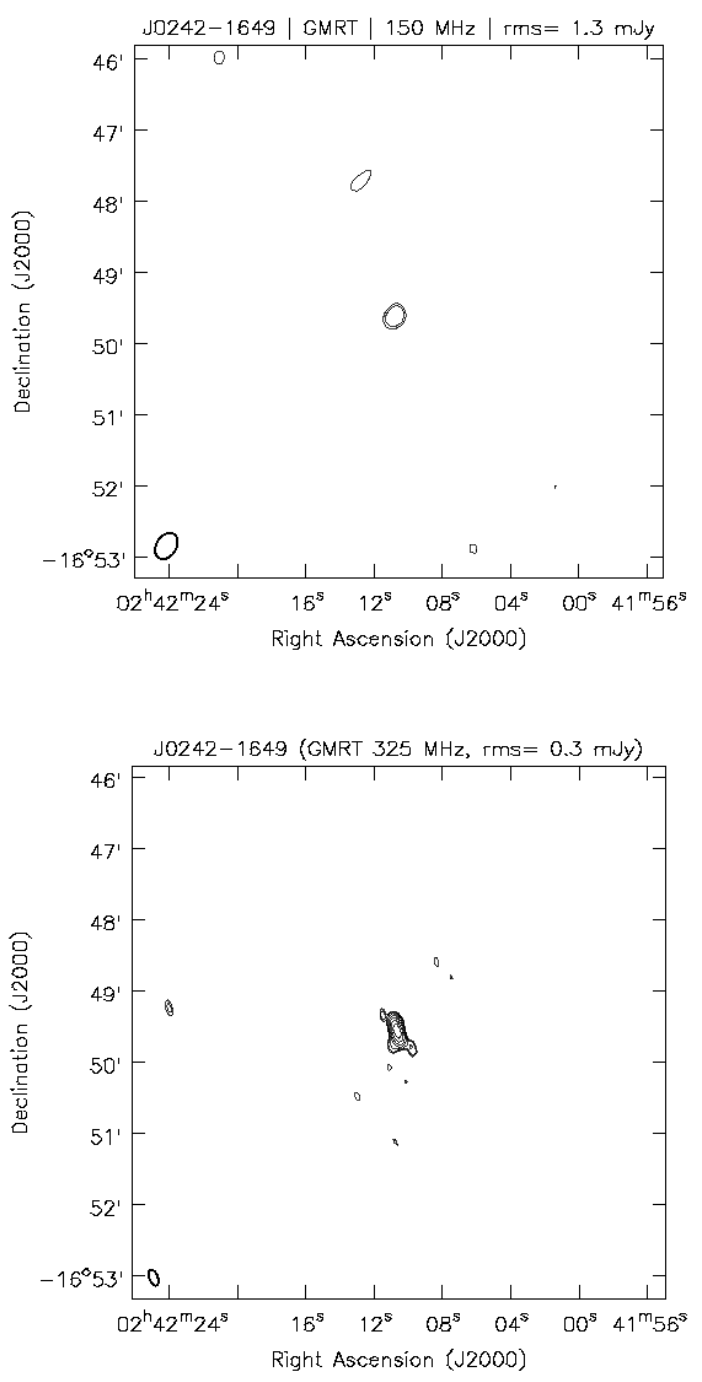

Figure 1. GMRT contour maps of J0242-1649 at $150 \mathrm{MHz}$ and $325 \mathrm{MHz}$, respectively. The contours are drawn at $3,4,8,16,32,64$ \& 128 times the image rms noise which is $1.3 \mathrm{mJy}$ at $150 \mathrm{MHz}$ and $0.3 \mathrm{mJy}$ at $325 \mathrm{MHz}$. The beam sizes are $23.7 \times 16.7 "(\mathrm{PA}=$ $\left.-33^{\circ}\right)$ and $14.1 \times 7.0^{\prime \prime}\left(\mathrm{PA}=-20^{\circ}\right)$ at $150 \mathrm{MHz}$ and $325 \mathrm{MHz}$, respectively. The target source lies at the centre of the map.

\section{J0442-1826}

The source is detected in the present $150 \mathrm{MHz}$ GMRT map $(\sigma \sim 1.2 \mathrm{mJy}$, Figure 2-online material), precisely at its NVSS and GMRT $325 \mathrm{MHz}$ positions (Table 2). However, the present flux density estimate at $150 \mathrm{MHz}(35.6 \mathrm{mJy})$ is approximately twice that reported in the TGSS/DR5 (see Paper I). Curiously, no source is detected at this position in the TGSS-ADR1 map at $150 \mathrm{MHz}(\sigma \sim 3.2 \mathrm{mJy}$, Figure 1online material). This is intriguing, particularly as all other sources of similar (and, of course higher) intensities have been detected in the two $150 \mathrm{MHz}$ maps just mentioned, as is indeed expected. Unfortunately, the source is too weak to appear in the GLEAM map at $151 \mathrm{MHz}(\sigma \sim 24 \mathrm{mJy})$. 
At $325 \mathrm{MHz}$, the present estimate of flux density (Table 2) is only marginally lower than the WISH value at $352 \mathrm{MHz}$ used in Paper I. The VLBI images at 4.3 and $8.4 \mathrm{GHz}$ reveal a resolved structure consisting of a dominant flat-spectrum and a linear extension of size $\sim 20$ mas towards south-east direction ${ }^{2}$.

\section{J1003-2514}

With a flux density of $9.4 \pm 3.4 \mathrm{mJy}$ this source is detected at just $2.8 \sigma$ level in the present $150 \mathrm{MHz}$ GMRT map (Figure 3-online material). In the $150 \mathrm{MHz}$ TGSS-ADR1 map ( $\sigma$ $\sim 4.1 \mathrm{mJy}$ ) it is registered at a stronger level of $19.2 \mathrm{mJy}$. Note that the flux scales of the two maps are found to be in good agreement (Table 3). In any case, with a spectral slope of $+2.11 \pm 0.50$ (Table 3 ), this source remains an interesting candidate to follow-up. We also note that its flux density of $143 \pm 6.1 \mathrm{mJy}$ in the WISH catalogue at $352 \mathrm{MHz}$, which was used in Paper I, is nearly a factor of 3 higher than the present estimate of $47.9 \pm 4.8 \mathrm{mJy}$ at $325 \mathrm{MHz}$. The large discrepancy could partly be due to uncertainty in the gain and absolute flux density calibrations, which is already over $10 \%$ for the WENSS (Hardcastle et al. 2016) and probably much more for WISH owing to its highly elongated beam. Since its nearest feature seen in the present $325 \mathrm{MHz}$ GMRT map, which is offset towards north-west by $\sim 1.5$ arcmin and is $\sim 4$ times weaker than the target source (Figure 3-online material), any confusion due to it is unlikely to account for the huge excess of the WISH flux density.

\section{J1031-2228}

The source is clearly seen in the present $150 \mathrm{MHz}$ GMRT map ( $\sigma \sim 3.1 \mathrm{mJy}$, Figure 4-online material). However, its flux density $(22.8 \pm 3.6 \mathrm{mJy})$ is much below the TGSSADR1 value of $56.1 \pm 9.5$ at $150 \mathrm{MHz}$ (Table 2). We note that the flux scale of our $150 \mathrm{MHz}$ map is consistent with the GLEAM map to within $\sim 2 \%$, but lower by $\sim 25 \%$ in comparison to the $150 \mathrm{MHz}$ TGSS-ADR1 map. However, even this falls too short of explaining the discrepancy. The large downward revision of flux density at $150 \mathrm{MHz}$ is mirrored when the present value of $\sim 107.1 \pm 10.7 \mathrm{mJy}$ at 325 $\mathrm{MHz}$ is compared with the WISH estimate of $191 \pm 7.1 \mathrm{mJy}$ at $325 \mathrm{MHz}$ which we used in Paper I. A similar, albeit less pronounced, trend is seen at $1.4 \mathrm{GHz}$, when the present estimate of $307.7 \mathrm{mJy}$ is compared with the NVSS value of 371.4 mJy. Taylor et al. (2009) have inferred a rotation measure of $52.6 \pm 18 \mathrm{rad} \mathrm{m}^{-2}$ for this source, suggesting a moderate presence of magneto-ionic plasma. The AT20G catalogue reports fractional polarisation of $4.8 \%$ at $20 \mathrm{GHz}, 2.1 \%$ at 8 $\mathrm{GHz}$ and $1.6 \%$ at $5 \mathrm{GHz}$ (Murphy et al. (2010)). The VLBA maps at 2.3, 4.3 and $7.6 \mathrm{GHz}$ exhibit a roughly linear structure of overall size $\sim 20$ mas, including a flat-spectrum central core and two lobes each resolved into peaks, the western lobe being the brighter one ${ }^{3}$.

2 http://astrogeo.org/vlbi_images/

3 http://astrogeo.org/vlbi_images/

\section{$\mathrm{J} 1207-2446$}

Unfortunately, its present GMRT $150 \mathrm{MHz}$ observations were severely affected by the RFI and therefore no useful map could be obtained. Nonetheless, an adequately sensitive TGSS-ADR1 map is available at this frequency $(\sigma \sim$ $3.6 \mathrm{mJy}$, Figure 5-online material), clearly showing the target source. A comparison with the GLEAM $150 \mathrm{MHz}$ map shows that the TGSS-ADR1 flux densities are systematically low by a factor FSF $\sim 1.6$ (Table 3 ). However, even if this is ignored, the source's spectral index is only $(\alpha=$ $1.17 \pm 0.21$, i.e., too small to qualify it as an EISERS (Table 2). Its present estimate at $325 \mathrm{MHz}$ is $\sim 172.3 \pm 17.2$ mJy which is only about half the WISH value we had used in Paper I. This has led to a major downward revision of its spectral index and the source is no longer an EISERS candidate.

\section{J1209-2032}

The present $150 \mathrm{MHz}$ GMRT map is comparatively noisy $(\sigma \sim 6.5 \mathrm{mJy}$, Figure 2$)$ near the field centre, yielding an upper limit of $19.5 \mathrm{mJy}$ for this undetected source. A further revision of this limiting value to $\sim 27 \mathrm{mJy}$ may be necessary since we find for this GMRT map a scaling factor of $\sim 1.4$, relative to both TGSS-ADR 1 and GLEAM maps (Table 3). While this upper limit at $150 \mathrm{MHz}$ is fully consistent with that given in Paper I, a large difference exists at $325 \mathrm{MHz}$, in the sense that the present GMRT estimate of $149 \pm 14.9 \mathrm{mJy}$ is significantly below the WISH catalog value of $207 \pm 8.4 \mathrm{mJy}$ at $352 \mathrm{MHz}$ which we used in $\mathrm{Pa}$ per I. The cause of this discrepancy is likely to be the same as mentioned above for the case of J1003-2514; it could be related to the presence of a few strong sources within the field (Figure 2) and the rather large, elongated beam of the WISH map (sect. 1). Using just the present GMRT flux densities at $150 \mathrm{MHz}$ and $325 \mathrm{MHz}$, gives a spectral index of $>+2.64 \pm 0.13$, making this source a possible case of EISERS. The other two estimates, $\alpha_{(1)} \& \alpha_{(2)}$ (Table 3) are somewhat smaller, and hence could well be consistent with the SSA limit. Healey et al. (2008) have identified this source with a galaxy at $z=0.404$. The AT20G catalogue reports fractional polarisation of $1 \%$ at $20 \mathrm{GHz}, 8 \mathrm{GHz}$ and $5 \mathrm{GHz}$ (Murphy et al. (2010)). The VLBI images at 2.3, 4.3 and $7.6 \mathrm{GHz}$ reveal a pair of lobes separated by $\sim 25$ mas, and straddling a flat-spectrum core ${ }^{4}$.

\section{J1626-1127}

The source is clearly detected in the present $150 \mathrm{MHz}$ GMRT map ( $\sigma \sim 7.5 \mathrm{mJy}$, Figure 6-online material), with a flux density of $37.8 \pm 8.4 \mathrm{mJy}$. This is consistent with the TGSS/DR5 value used in Paper I. Unlike the present 150 $\mathrm{MHz}$ GMRT map, the source is seen to have some resolved emission in the TGSS-ADR 1 map $(\sigma=3.3 \mathrm{mJy})$ and has a much higher integrated flux density $(\sim 84.1 \pm 9.5 \mathrm{mJy}$ at $150 \mathrm{MHz}$ ). No other discrepancy is evident between the two maps, except for the flux scaling factor which is somewhat large (FSF $\sim 1.26$, Table 2). At $325 \mathrm{MHz}$, the source

${ }^{4}$ http://astrogeo.org/vlbi_images/ 

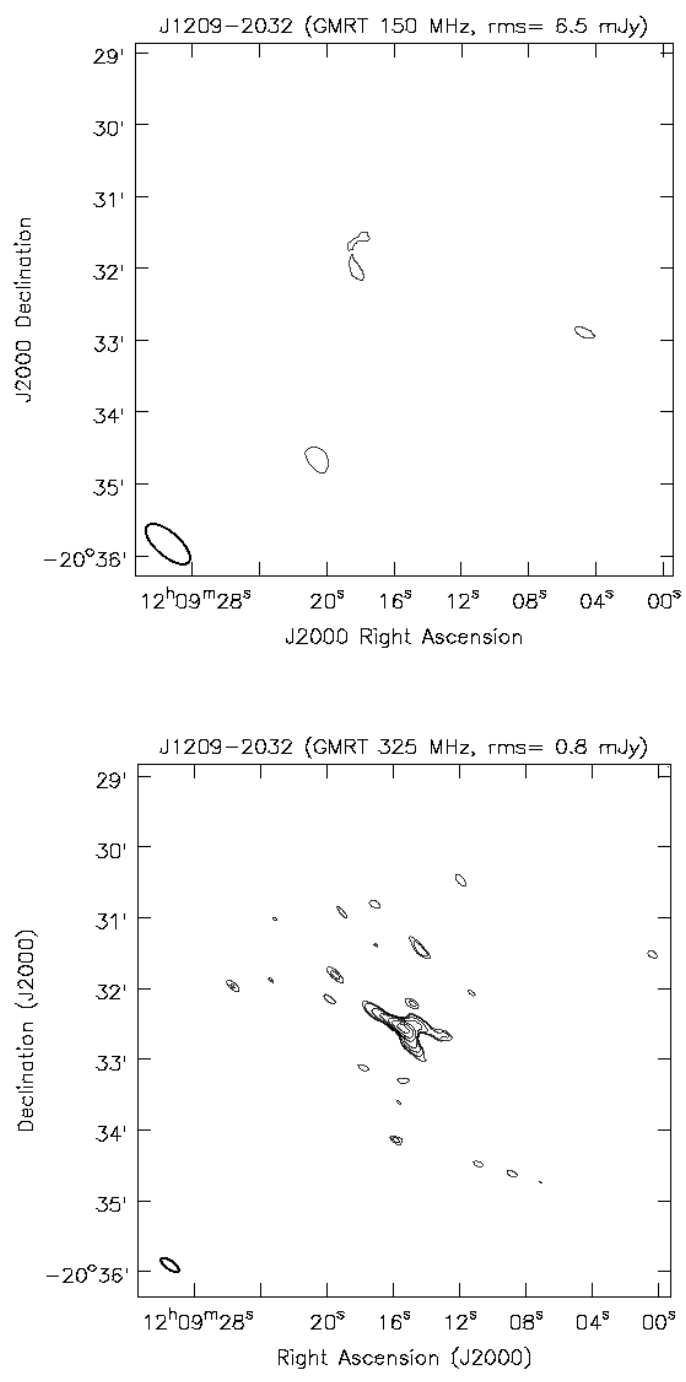

Figure 2. GMRT contour maps of J1209-2032 at $150 \mathrm{MHz}$ and $325 \mathrm{MHz}$, respectively. The contours are drawn at $3,4,8,16,32,64$ and 128 times the image rms noise which is $6.5 \mathrm{mJy}$ at $150 \mathrm{MHz}$ and $0.8 \mathrm{mJy}$ at $325 \mathrm{MHz}$. The beam sizes are $31.9 \times 13.4 "(\mathrm{PA}=$ $\left.47^{\circ}\right)$ and $17.7 \times 7.1^{\prime \prime}\left(\mathrm{PA}=54^{\circ}\right)$ at $150 \mathrm{MHz}$ and $325 \mathrm{MHz}$, respectively. The target source lies at the centre of the map. The complex structure seen at $325 \mathrm{MHz}$ map probably suffers from severe artefacts, arising from the exceptionally short duration (5 mins) for which this source could be observed (Table 1).

is seen at a significantly lower level in the present GMRT map (152.5 $\pm 15.3 \mathrm{mJy})$, as compared to the WISH value of $206 \pm 8.5 \mathrm{mJy}$ at $352 \mathrm{MHz}$ which we used in Paper I. But in either case, its spectral index remains below +2.0 (Table 2), ruling it out as an EISERS candidate.

\section{DISCUSSION}

Based on the estimates of two-frequency spectral index $\alpha(150-325 \mathrm{MHz})$ (Table 2), we confirm J0242-1649 with $\alpha=+2.95 \pm 0.31$ to be a bona-fide EISERS. Another source, J1209-2032 having $\alpha>+2.64 \pm 0.13$ is a promising EISERS candidate, i.e., $\alpha$ marginally above the SSA limit of $\alpha_{c}=$ +2.5 . None of the remaining 5 target sources is found to approach $\alpha_{c}$, although J1003-2514 and J1031-2228 do have very steeply inverted spectra, with $\alpha(150-325 \mathrm{MHz}) \sim+2.0$. To verify if they are genuine EISERS, spectral measurements below $150 \mathrm{MHz}$ will be helpful. It is encouraging that the two best cases of EISERS, emerging from the present work, are the same two that we had highlighted in Paper I.

Recently, CEG17 have reported a few promising examples of EISERS based on the GLEAM survey which has catalogued nearly $10^{5}$ unresolved radio sources south of declination $+30 \mathrm{deg}$ and above a flux density limit of $0.16 \mathrm{Jy}$ at $200 \mathrm{MHz}$, the effective frequency of the 'deep wide-band images' formed by integrating over the 170-231 MHz bandwidth (Hurley-Walker et al. 2017; Wayth et al. 2015). Note that the frequency of the present GMRT observations (and also of the TGSS-ADR1 (Intema et al. 2017)) is almost a perfect match to the central frequency of the GLEAM survey $(151 \mathrm{MHz})$. The key merit of the GLEAM survey is that it provides for each source essentially contemporaneous flux density measurements at several frequencies in the range 72 $-231 \mathrm{MHz}$. The angular resolution, however, is modest ( $2 \operatorname{arcmin}$ at $200 \mathrm{MHz}$ ) and, more importantly, the rms noise is usually much higher, starting with $\sim 20 \mathrm{mJy} /$ beam at $231 \mathrm{MHz}$ and rising to $\sim 100 \mathrm{mJy}$ at $72 \mathrm{MHz}$. This latter point is specially pertinent for the discussion below, where we compare the findings of CEG17 with the present results.

Table 3 of CEG17 lists six radio sources for which their estimates of spectral index $\left(\alpha_{\text {thick }}\right)$ in the optically thick region are close to, or exceed the SSA limit of $\alpha_{c}=+2.5$. For two of them (J144815-162024 and J211949-343936), $\alpha_{\text {thick }}$ is actually consistent with the SSA limit of +2.5 (Table 3 ). For another source, J001513-472706, the GLEAM measurements at the lowest frequencies are too noisy to effectively constrain its spectral slope in the optically thick region. Likewise, difficulty arises for the source J100256-354157 because of the poor fit of the theoretical spectrum to the flux measurements. For the remaining two sources, J074211-673309 and J213024-434819, CEG17 have estimated $\alpha_{\text {thick }}$ to be $4.1 \pm 0.9$ and $3.2 \pm 0.6$, respectively. One aught to appreciate the model dependent nature of these estimates, since they actually represent the terminal slope of the theoretical synchrotron spectrum of a uniform synchrotron source (Eqn. 3 of CEG17), which CEG17 have fitted to their spectral measurements between 72 to $843 / 1400$ $\mathrm{MHz}$ (note also that the points at 843 and $1400 \mathrm{MHz}$ actually come from the observations made more than a decade prior to the GLEAM survey which only covers the $72-231$ $\mathrm{MHz}$ band).

In view of this, we have independently determined for the above two sources the slope of the inverted radio spectrum, by a weighted least-square fitting of a straight line to the GLEAM flux density measurements at their lowest (up to) 4 frequencies (since the effect of SSA is expected to be most pronounced at the lowest frequencies). These computed values, given in Table 4, show that for J074211-673309, the uncertainty in the slope of the fitted spectrum is too large to infer a violation of the SSA limit of $\alpha_{\text {thick }}=+2.5$. On the other hand, our best-fit spectral slope for J213024-434819 breaches the SSA limit by a significant 

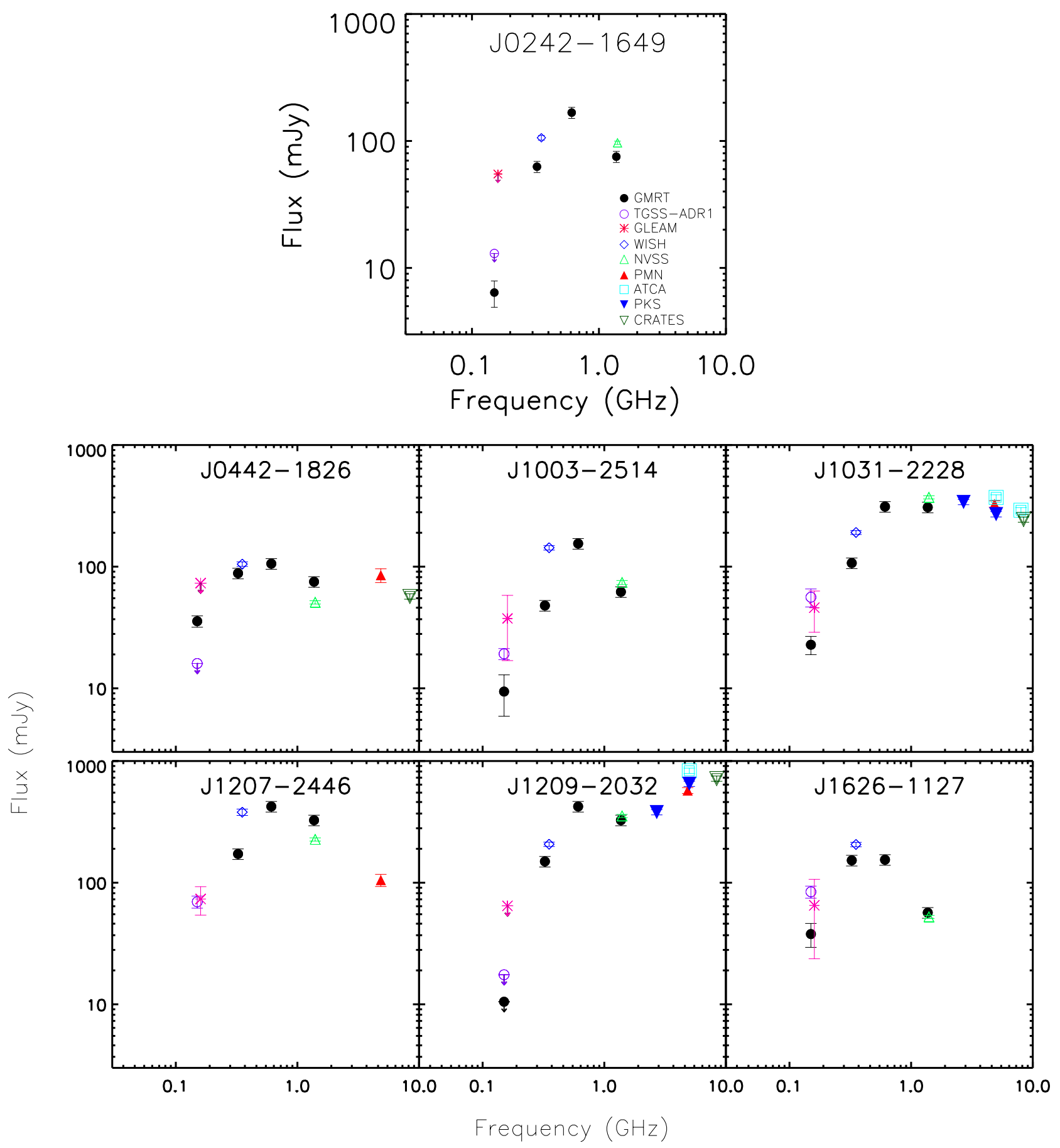

Figure 3. Radio spectra of the 7 target sources (see Table 2). In each panel, the names of the surveys are mentioned next to the corresponding symbol, with the frequency of the survey increasing downwards. The filled circles represent the measurements from the present GMRT observations at the 4 frequencies. 
Inverted spectrum extragalactic radio sources

Table 4. The GLEAM survey flux densities and the estimated spectral indices ${ }^{\star}$ for J074211-673309 and J213024-434819.

\begin{tabular}{lcc}
\hline Parameters & J074211-673309 & J213024-434819 \\
\hline $\mathrm{S}_{76}(\mathrm{mJy}) \dagger$ & $0.69 \pm 0.12$ & $0.15 \pm 0.07$ \\
$\mathrm{~S}_{84}(\mathrm{mJy})$ & $0.89 \pm 0.10$ & $0.27 \pm 0.05$ \\
$\mathrm{~S}_{92}(\mathrm{mJy})$ & $1.02 \pm 0.09$ & $0.38 \pm 0.05$ \\
$\mathrm{~S}_{99}(\mathrm{mJy})$ & $1.14 \pm 0.10$ & $0.52 \pm 0.05$ \\
$\alpha_{(1)}$ & $2.54 \pm 2.06$ & $5.70 \pm 4.79$ \\
$\alpha_{(2)}$ & $1.89 \pm 0.34$ & $4.28 \pm 0.60$ \\
$\alpha_{(3)}$ & $1.70 \pm 0.19$ & $4.26 \pm 0.23$ \\
\hline
\end{tabular}

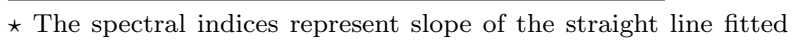
to the flux densities (and errors) by the weighted least-square method (see text).

$\dagger$ Flux densities at 76, 84, 92 and $99 \mathrm{MHz}$, taken from the GLEAM survey (CEG17).

$\alpha_{(1)}$ is the spectral index, based on the fit to the flux densities at the lowest two frequencies (sect. 5).

$\alpha_{(2)}$ is the spectral index, based on the fit to the flux densities at the lowest three frequencies (sect. 5).

$\alpha_{(3)}$ is the spectral index, based on the fit to the flux densities at the lowest four frequencies (sect. 5).

margin, in accord with the claim of CEG17 (although they favour the FFA interpretation for the spectral turnover, on statistical ground).

Here it may be cautioned that the present linear fitting to the GLEAM data points is not strictly valid and is merely intended to identify the most promising EISERS candidates. The problem relates to the correlated nature of the data in the GLEAM sub-bands (see, Hurley-Walker et al. 2017), which means that the GLEAM measurements for individual sub-bands (such as the last four data points mentioned above) are not fully independent and, consequently, systematics can drive correlation and influence the measurements reproduced in Table 4 (potentially making a spectrum steeper or shallower). Bearing this in mind, if one assumes that the computed spectral slope of J213024-434819, which is based on the GLEAM measurement (Table 4, will be confirmed by independent observations, both J0242-1649 (present work; Paper I) and J213024-434819 remain the best EISERS candidates known. A third good candidate is J1209-2032 $(\alpha>2.64 \pm 0.13$ between 150 and $325 \mathrm{MHz})$ which is identified with a galaxy at $\mathrm{z}=0.404$ (Table 2). It does not have a GPS type radio spectrum, unlike J0242-1649 (Figure 3). As mentioned in sect. 4, its VLBI observations at $2.3 \mathrm{GHz}$ show it to be a very young radio source, with a total extent of only 25 msec (i.e., $\sim 140$ parsec at $\mathrm{z}=0.404)$. For such young radio sources, the observed sharp spectral turnover at metre wavelengths is also consistent with FFA occurring in a warm inhomogeneous interstellar medium plausibly associated with the host elliptical galaxy (Bicknell et al. 2018).

\section{CONCLUSIONS}

This study presents our continued search for extragalactic sources whose radio continuum spectrum is so sharply inverted due to opacity effects that its slope $\alpha$ exceeds +2.5 , thus becoming inconsistent with the standard interpretation in terms of synchrotron self-absorption (SSA) of the incoherent synchrotron radiation arising from relativistic particles with a power-law energy distribution. Here, we have reported sensitive, quasi-simultaneous GMRT observations at $150,325,610$ and $1400 \mathrm{MHz}$ of the seven sources we had previously identified as candidates for inverted radio spectrum which is steepened beyond the SSA limit (viz., EISERS). However, that surmise was based on published multifrequency radio observations that are only moderately sensitive, as well as highly non-contemporaneous. Addressing both these shortcomings, our targeted GMRT observations at the four frequencies, as reported here, show that for at least one (probably, two) of the seven candidates, the spectral index computed between the lowest two frequencies, exceeds the SSA limit of $\alpha=+2.5$. This, together with another one or two extragalactic sources that are reported in very recent literature to exhibit a similarly extreme radio spectrum (sect. 5; Table 4), raises the possibility that in some very rare cases, the SSA limit may be violated. However, if future observations indicate the presence of dense thermal plasma towards these extragalactic sources, that would render free-free absorption as a less radical and hence perhaps more attractive alternative explanation for the ultra-sharp turnover observed in their radio spectra. Search for additional sources with such extremely inverted radio continuum spectra forms a key objective of our ongoing programme, backed up with an ongoing follow-up using the upgraded GMRT (Gupta et al. 2017).

\section{ACKNOWLEDGEMENTS}

The Giant Metrewave Radio Telescope (GMRT) is a national facility operated by the National Centre for Radio Astrophysics (NCRA) of the Tata Institute of Fundamental Research (TIFR). We thank the staff at NCRA and GMRT for their support. This research has used the TIFR. GMRT. Sky. Survey (http://tgss.ncra.tifr.res.in) data products, NASA's Astrophysics Data System and NASA/IPAC Extragalactic Database (NED), Jet Propulsion Laboratory, California Institute of Technology under contract with National Aeronautics and Space Administration and VizieR catalogue access tool, CDS, Strasbourg, France. We thank the NRAO staff for providing AIPS. G-K thanks the National Academy of Sciences, India for the award of a NASI Senior Scientist Platinum Jubilee Fellowship. PD gratefully acknowledges generous support from the Indo-French Center for the Promotion of Advanced Research (Centre FrancoIndien pour la Promotion de la Recherche Avanćee) under programme no. 5204-2 and IUCAA for its computational facilities.SP wants to thank DST INSPIRE Faculty Scheme (IF12/PH-44) for funding his research group. The authors would like to thank the anonymous referee for the valuable suggestions.

\section{REFERENCES}

An T., Baan W. A., 2012, ApJ, 760, 77

Athreya R. M., Kapahi V. K., 1998, Journal of Astrophysics and Astronomy, 19, 63

Baars J. W. M., Genzel R., Pauliny-Toth I. I. K., Witzel A., 1977, A\&A, 61, 99 
Bell M. E., et al., 2019, MNRAS, 482, 2484

Bicknell G. V., Dopita M. A., O'Dea C. P. O., 1997, ApJ, 485, 112

Bicknell G. V., Mukherjee D., Wagner A. Y., Sutherland R. S., Nesvadba N. P. H., 2018, MNRAS, 475, 3493

Callingham J. R., et al., 2015, ApJ, 809, 168

Callingham J. R., et al., 2017, ApJ, 836, 174

Chandra P., Ray A., Bhatnagar S., 2004, ApJ, 612, 974

Condon J. J., Cotton W. D., Greisen E. W., Yin Q. F., Perley R. A., Taylor G. B., Broderick J. J., 1998, AJ, 115, 1693

Coppejans R., Cseh D., Williams W. L., van Velzen S., Falcke H., 2015, MNRAS, 450, 1477

Coppejans R., et al., 2016, MNRAS, 459, 2455

Dallacasa D., Stanghellini C., Centonza M., Fanti R., 2000, A\&A, 363,887

De Breuck C., Tang Y., de Bruyn A. G., Röttgering H., van Breugel W., 2002, A\&A, 394, 59

Falcke H., et al., 1999, ApJ, 514, L17

Falcke H., Körding E., Nagar N. M., 2004, New Astron. Rev., 48, 1157

Gopal-Krishna Spoelstra T. A. T., 1993, A\&A, 271, 101

Gopal-Krishna Patnaik A. R., Steppe H., 1983, A\&A, 123, 107

Gopal-Krishna Mhaskey M., Mangalam A., 2012, ApJ, 744, 31

Gopal-Krishna Sirothia S. K., Mhaskey M., Ranadive P., Wiita P. J., Goyal A., Kantharia N. G., Ishwara-Chandra C. H., 2014, MNRAS, 443, 2824

Griffith M. R., Wright A. E., Burke B. F., Ekers R. D., 1994, ApJS, 90, 179

Gupta Y., et al., 2017, Current Science, 113, 707

Hardcastle M. J., et al., 2016, MNRAS, 462, 1910

Healey S. E., et al., 2008, ApJS, 175, 97

Hurley-Walker N., et al., 2017, MNRAS, 464, 1146

Intema H. T., 2014, in Astronomical Society of India Conference Series. (arXiv:1402.4889)

Intema H. T., Jagannathan P., Mooley K. P., Frail D. A., 2017, A\&A, 598, A78

Jones D. L., et al., 1996, ApJ, 466, L63

Jones D. L., Wehrle A. E., Piner B. G., Meier D. L., 2001, ApJ, 553,968

Kadler M., Ros E., Lobanov A. P., Falcke H., Zensus J. A., 2004, A\&A, 426, 481

Kameno S., Horiuchi S., Shen Z.-Q., Inoue M., Kobayashi H., Hirabayashi H., Murata Y., 2000, PASJ, 52, 209

Kardashev N. S., 1962, Soviet Ast., 6, 317

Kellermann K. I., 1964, ApJ, 140, 969

Kellermann K. I., 1966, Australian Journal of Physics, 19, 195

Kellermann K. I., Pauliny-Toth I. I. K., 1969, ApJ, 155, L71

Klamer I. J., Ekers R. D., Bryant J. J., Hunstead R. W., Sadler E. M., De Breuck C., 2006, MNRAS, 371, 852

Krichbaum T. P., Alef W., Witzel A., Zensus J. A., Booth R. S., Greve A., Rogers A. E. E., 1998, A\&A, 329, 873

Kuncic Z., Bicknell G. V., Dopita M. A., 1998, ApJ, 495, L35

Levinson A., Laor A., Vermeulen R. C., 1995, ApJ, 448, 589

Mangalam A. V., Gopal-Krishna 1995, MNRAS, 275, 976

Matveenko L. I., Pauliny-Toth I. I. K., Sherwood W., 1990, Soviet Astronomy Letters, 16, 247

Murgia M., Fanti C., Fanti R., Gregorini L., Klein U., Mack K.H., Vigotti M., 2002, New Astron. Rev., 46, 307

Murphy T., et al., 2010, MNRAS, 402, 2403

O'Dea C. P., 1998, PASP, 110, 493

Ostorero L., et al., 2010, ApJ, 715, 1071

Pacholczyk A. G., 1970, Radio astrophysics. Nonthermal processes in galactic and extragalactic sources

Perley R. A., Butler B. J., 2017, ApJS, 230, 7

Rees M. J., 1967, MNRAS, 136, 279

Rengelink R. B., Tang Y., de Bruyn A. G., Miley G. K., Bremer M. N., Roettgering H. J. A., Bremer M. A. R., 1997, A\&AS, 124,259
Roger R. S., Costain C. H., Bridle A. H., 1973, AJ, 78, 1030

Rybicki G. B., Lightman A. P., 1986, Radiative Processes in Astrophysics

Scheuer P. A. G., Williams P. J. S., 1968, ARA\&A, 6, 321

Slish V. I., 1963, Nature, 199, 682

Spoelstra T. A. T., Patnaik A. R., Gopal-Krishna 1985, A\&A, 152,38

Stawarz L., Ostorero L., Begelman M. C., Moderski R., Kataoka J., Wagner S., 2008, ApJ, 680, 911

Swarup G., 1991, in Cornwell T. J., Perley R. A., eds, Astronomical Society of the Pacific Conference Series Vol. 19, IAU Colloq. 131: Radio Interferometry. Theory, Techniques, and Applications. pp 376-380

Taylor A. R., Stil J. M., Sunstrum C., 2009, ApJ, 702, 1230

Tingay S. J., Murphy D. W., 2001, ApJ, 546, 210

Tingay S. J., de Kool M., 2003, AJ, 126, 723

Vermeulen R. C., Ros E., Kellermann K. I., Cohen M. H., Zensus J. A., van Langevelde H. J., 2003, A\&A, 401, 113

Walker R. C., Dhawan V., Romney J. D., Kellermann K. I., Vermeulen R. C., 2000, ApJ, 530, 233

Wayth R. B., et al., 2015, Publ. Astron. Soc. Australia, 32, e025 de Kool M., Begelman M. C., 1989, Nature, 338, 484

van Breugel W., 1984, in Fanti R., Kellermann K. I., Setti G., eds, IAU Symposium Vol. 110, VLBI and Compact Radio Sources. p. 59 
This paper has been typeset from a $\mathrm{T}_{\mathrm{E}} \mathrm{X} / \mathrm{LAT} \mathrm{T}$. file prepared by the author.

MNRAS 000, 1-11 (2018) 


\title{
GMRT observations of extragalactic radio sources with steeply inverted spectra
}

\author{
Mukul Mhaskey ${ }^{1}$, Gopal-Krishna ${ }^{2}$, Pratik Dabhade ${ }^{3,4}$, Surajit Paul $^{1}$ \\ Sameer Salunkhe ${ }^{1}$, and S.K. Sirothia ${ }^{5,6}$

\footnotetext{
${ }^{1}$ Department of Physics, Savitribai Phule Pune Unversity, Ganeshkhind, Pune 411007, India

2 Aryabhatta Research Institute of Observational Sciences (ARIES), Manora Peak, Nainital - 263129, India

${ }^{3}$ Inter University Centre for Astronomy and Astrophysics (IUCAA), Pune 411007, India

${ }^{4}$ Leiden Observatory, Leiden University, Niels Bohrweg 2, 2333 CA, Leiden, Netherlands

${ }^{5}$ Square Kilometre Array South Africa, 3rd Floor, The Park, Park Road, Pinelands, 7405, South Africa
} \\ ${ }^{6}$ Department of Physics and Electronics, Rhodes University, PO Box 94, Grahamstown, 6140, South Africa
}
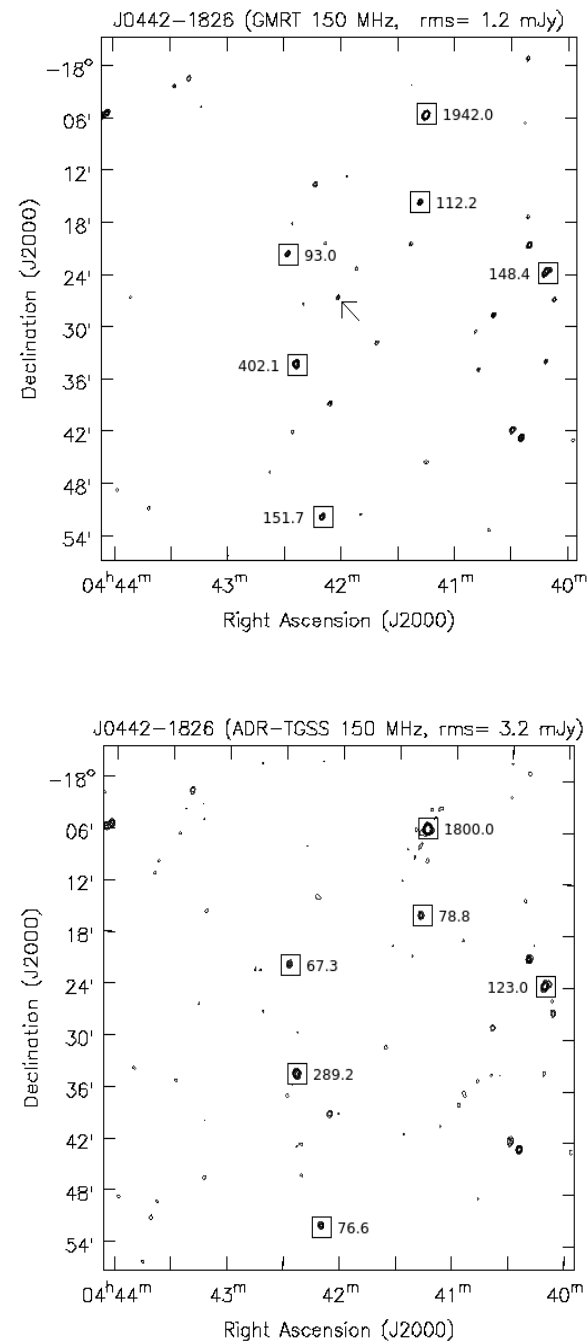

Figure 1: Contour maps of J0442-1649 at $150 \mathrm{MHz}$ from the present GMRT observations (upper panel) and the TGSS-ADR1 (lower panel). The contours in both the maps are plotted in the intensity units $(\mathrm{mJy} / \mathrm{beam})$ for comparison sake. The relative contour levels are $0.005,0.010,0.0 .020,0.040,0.080,0.160,0.320$ with the unit contour set to 1 . The contours start at $5 \mathrm{mJy}$ and double every level. The target source J0442-1649 ( 35.6 mJy, Table 2) is seen at the centre of the present GMRT map (marked with arrow); however it is undetected in the ADR-TGSS map. The beam size is $27.2 \times 17.4^{\prime \prime}\left(\mathrm{PA}=-34^{\circ}\right)$ at $150 \mathrm{MHz}(\mathrm{GMRT})$ and 31.83 $\times 25.0^{\prime \prime}$ at $150 \mathrm{MHz}$ (TGSS-ADR1). The target source J0442$1649(\sim 35.6 \mathrm{mJy}$, Table 2$)$ is seen at the centre of the present GMRT map; however it is undetected in the ADR-TGSS map. Flux densities (mJy), marked for several unresolved sources in the two maps, were used for calculating the flux scaling factor (FSF) between these maps (Table 3 ).
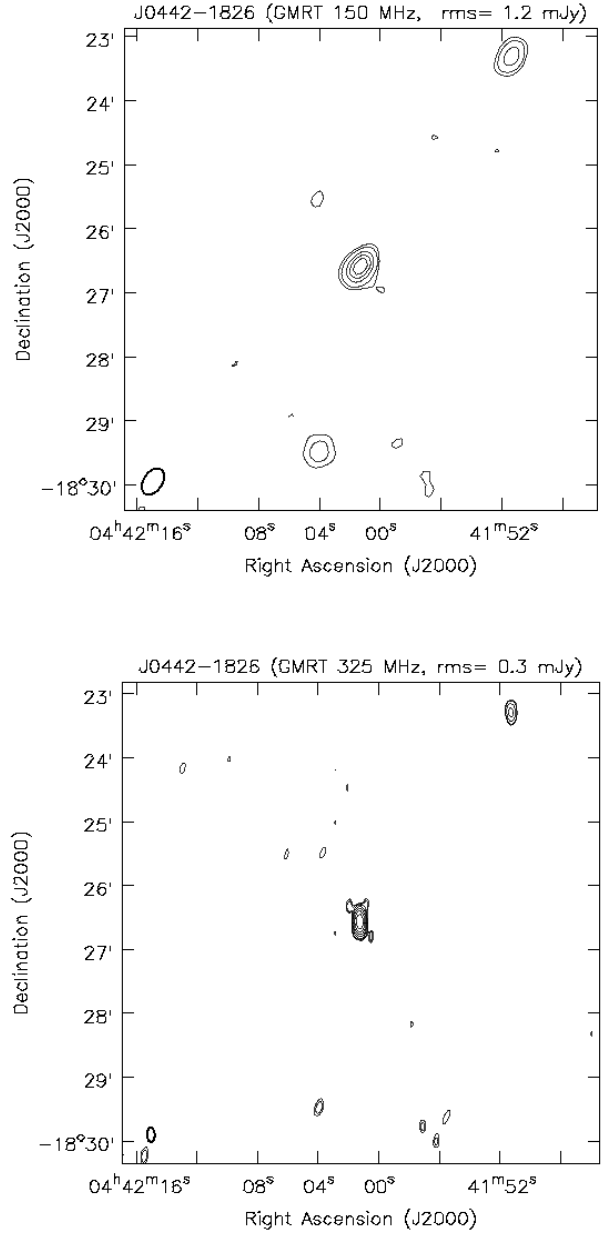

Figure 2: GMRT contour maps of J0442-1826 at $150 \mathrm{MHz}$ and $325 \mathrm{MHz}$, respectively. The contour levels are $3,4,8,16,32,64 \& 128$ with the unit contour level at $150 \mathrm{MHz}$ at $1.3 \mathrm{mJy}$ and $0.3 \mathrm{mJy}$ at $325 \mathrm{MHz}$. The beam size is $27.2 \times 17.4^{\prime \prime}\left(\mathrm{PA}=-34^{\circ}\right)$ and $13.7 \times 6.9^{\prime \prime}\left(\mathrm{PA}=2^{\circ}\right)$ at $150 \mathrm{MHz}$ and $325 \mathrm{MHz}$ respectively. The target source lies at the centre of the map. 

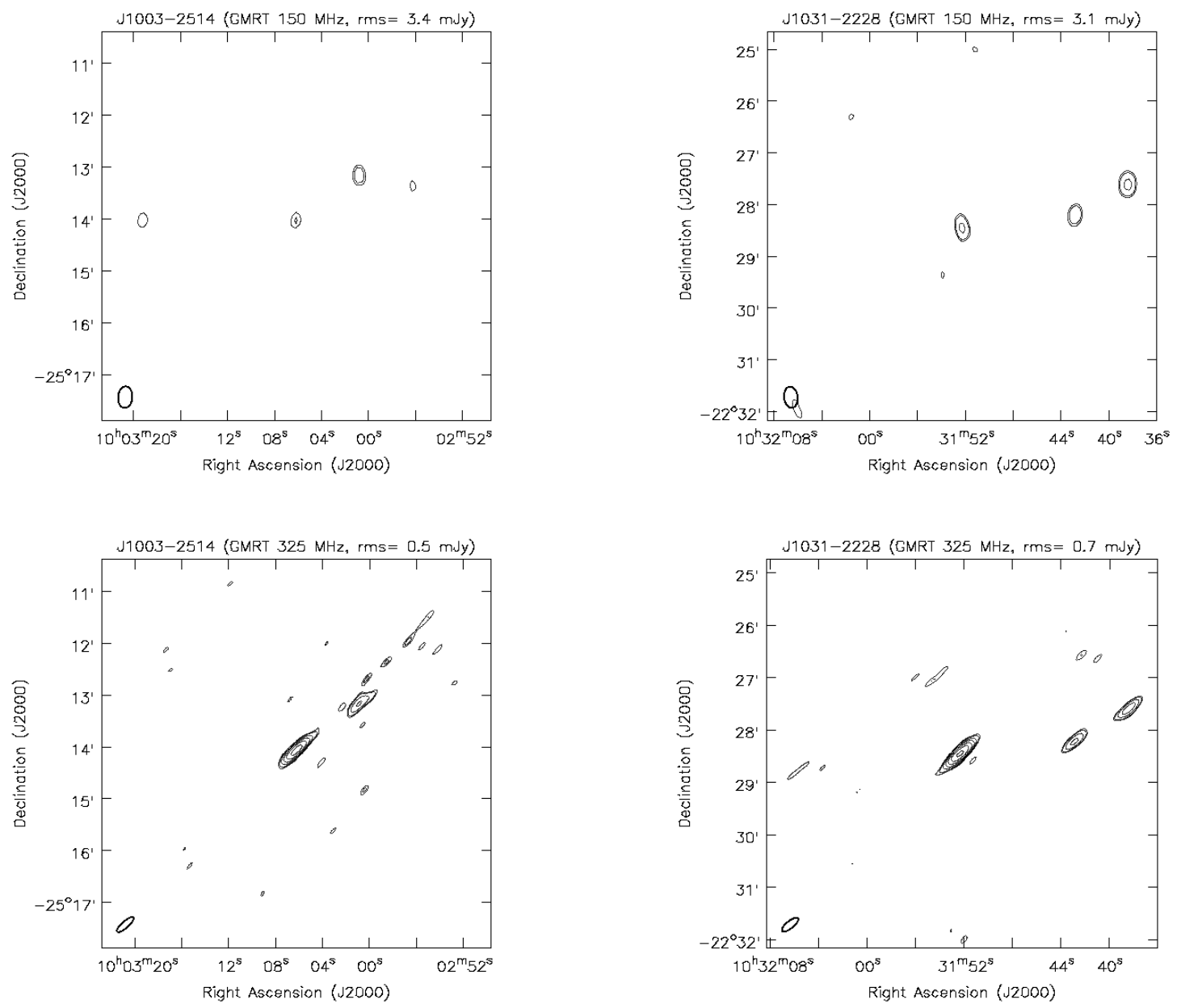

Figure 3: GMRT contour maps of J1003-2514 at $150 \mathrm{MHz}$ and $325 \mathrm{MHz}$, respectively. The contour levels are $3,4,8,16,32,64$ \& 128 with the unit contour level at $150 \mathrm{MHz}$ at $3.4 \mathrm{mJy}$ and 0.5 mJy at $325 \mathrm{MHz}$. The beam size is $24.9 \times 16.4^{\prime \prime}\left(\mathrm{PA}=-4^{\circ}\right)$ and $24.6 \times 7.6^{\prime \prime}\left(\mathrm{PA}=-48^{\circ}\right)$ at $150 \mathrm{MHz}$ and $325 \mathrm{MHz}$ respectively. The target source lies at the centre of the map.

Figure 4: GMRT contour maps of J1031-2228 at $150 \mathrm{MHz}$ and $325 \mathrm{MHz}$, respectively. The contour levels are $3,4,8,16,32,64$ \& 128 with the unit contour level at $150 \mathrm{MHz}$ at $3.1 \mathrm{mJy}$ and 0.7 mJy at $325 \mathrm{MHz}$. The beam size is $24.6 \times 15.7^{\prime \prime}(\mathrm{PA}=70)$ and $23.1 \times 8.2^{\prime \prime}\left(\mathrm{PA}=-51^{\circ}\right)$ at $150 \mathrm{MHz}$ and $325 \mathrm{MHz}$ respectively. The target source lies at the centre of the map. 

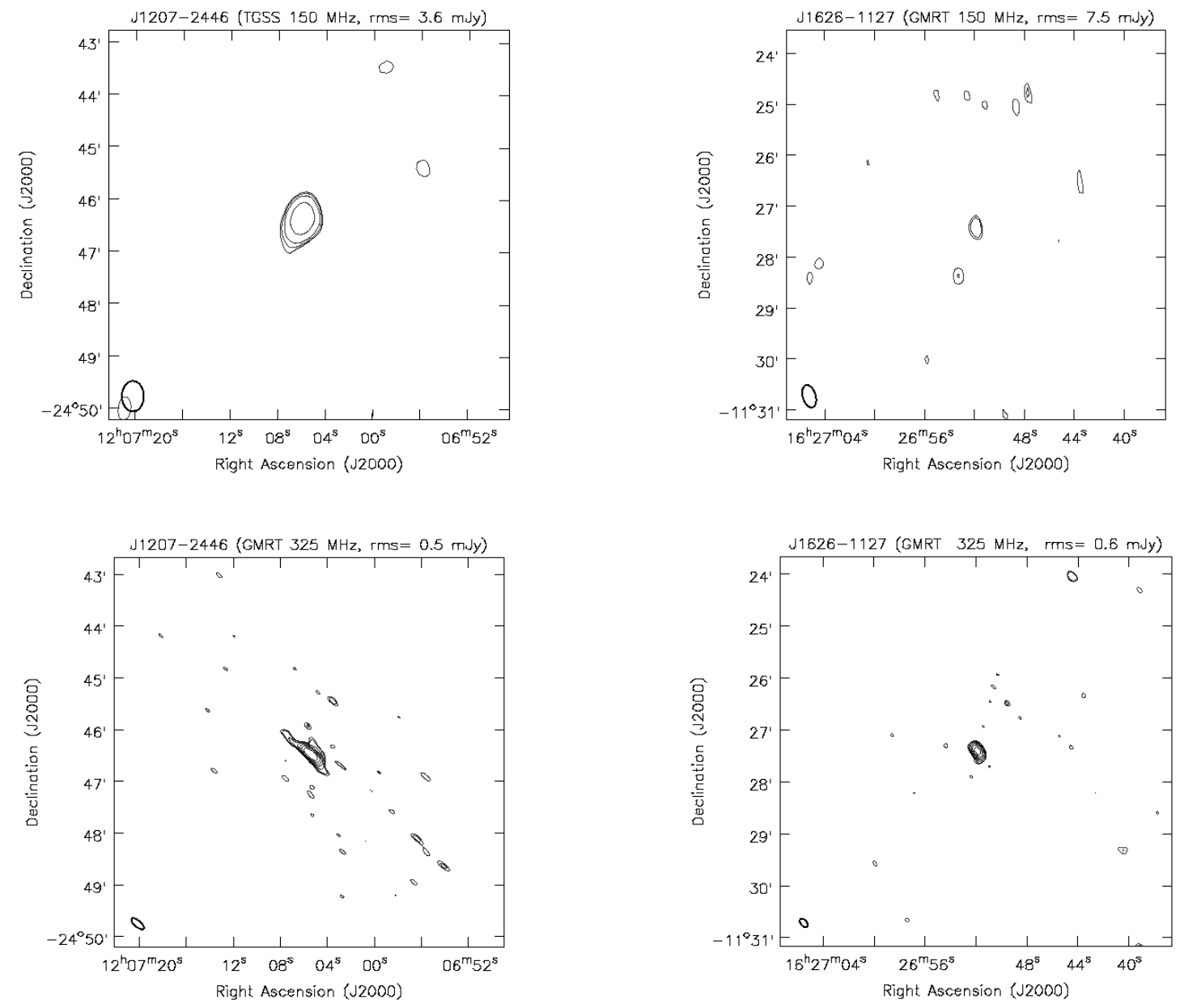

Figure 5: GMRT contour maps of J1207-2446 at $150 \mathrm{MHz}$ and $325 \mathrm{MHz}$, respectively. The contour levels are $3,4,8,16,32,64$ \& 128 with the unit contour level at $150 \mathrm{MHz}$ at $3.6 \mathrm{mJy}$ and 0.5 mJy at $325 \mathrm{MHz}$. The beam size is $33.7 \times 12.8^{\prime \prime}\left(\mathrm{PA}=42^{\circ}\right)$ and $18.1 \times 7.3 "\left(\mathrm{PA}=50^{\circ}\right)$ at $150 \mathrm{MHz}$ and $325 \mathrm{MHz}$ respectively. The target source lies at the centre of the map

Figure 6: GMRT contour maps of J1626-1127 at $150 \mathrm{MHz}$ and $325 \mathrm{MHz}$, respectively. The contour levels are $3,4,8,16,32,64$ \& 128 with the unit contour level at $150 \mathrm{MHz}$ at $7.5 \mathrm{mJy}$ and 0.6 mJy at $325 \mathrm{MHz}$. The beam size is $27.3 \times 15.1^{\prime \prime}\left(\mathrm{PA}=17^{\circ}\right)$ and $11.7 \times 6.8^{\prime \prime}\left(\mathrm{PA}=38^{\circ}\right)$ at $150 \mathrm{MHz}$ and $325 \mathrm{MHz}$ respectively. The target source lies at the centre of the map. 